\title{
An unexpected invasive hydatidiform mole in a rudimentary uterine horn: A case report
}

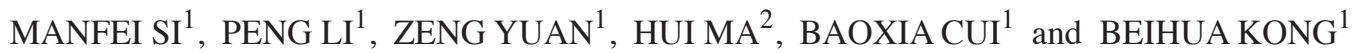 \\ ${ }^{1}$ Department of Obstetrics and Gynecology, Qilu Hospital of Shandong University, Jinan, Shandong 250012; \\ ${ }^{2}$ Department of Emergency, Affiliated Hospital of Shandong Academy of Medical Science, Jinan, Shandong 250031, P.R. China
}

Received August 21, 2015; Accepted January 11, 2017

DOI: $10.3892 / \mathrm{ol} .2017 .6493$

\begin{abstract}
Rudimentary horns of the uterus develop as a result of a partial non-development of one Müllerian duct, a type of congenital uterine anomaly. Pregnancy in a rudimentary horn is uncommon and the outcome tends to be poor, with the majority of cases resulting in rupture between 10 and 15 gestational weeks, with significant risk of morbidity and mortality. Regardless of the availability of imagiological procedures and the advances being made in this field, the diagnosis of this type of ectopic pregnancy often only occurs during laparotomy or laparoscopy subsequent to abdominal pain and collapse. The present study describes a rare case of a uterine rudimentary horn pregnancy. The case was diagnosed by the high serum $\beta$-HCG level, imageological results and dissection of the final pathological specimen, by combined hysteroscopy and laparoscopy. An ultrasound illustrated a suspicious gestational trophoblastic disease in the rudimentary uterine horn, with a rich blood flow signal at the right side of the uterus. A pelvic magnetic resonance image indicated that there was endometrial thickening and gestational trophoblastic disease in the rudimentary uterine horn. The patient was treated with surgery, including rudimentary horn dissection and diagnostic curettage; the ipsilateral adnexa was conserved as it appeared to be normal. Due to the apparent decline of the serum $\beta-\mathrm{HCG}$ level and the reluctance for chemotherapy, the patient chose to undergo no further treatment and closed follow-up. At the time of writing, the patient is in a good condition. Only a small number of reports of a horn pregnancy with invasive
\end{abstract}

Correspondence to: Professor Beihua Kong, Department of Obstetrics and Gynecology, Qilu Hospital, Shandong University, 107 West Wenhua Road, Jinan, Shandong 250012, P.R. China

E-mail: kongbeihua@sdu.edu.cn

Abbreviations: $\beta$-HCG, $\beta$-human chorionic gonadotropin; MTX, methotrexate; RHP, rudimentary horn pregnancy; MRI, magnetic resonance imaging; GTD, gestational trophoblastic disease; GTN, gestational trophoblastic neoplasia; IHM, invasive hydatidiform mole; CT, computed tomography

Key words: rudimentary horn of uterus, invasive hydatidiform mole, congenital uterine anomalies, ectopic gestation hydatidiform mole are described by other studies, thereby increasing the clinical significance of this case.

\section{Introduction}

Anatomical irregularities of the female genital tract resulting from abnormalities of the embryogenesis of the Müllerian duct system are relatively common. Between 1 in 200 and 1 in 600 fertile women are reported to be affected by congenital uterine anomalies, however, among these cases, rudimentary horns are rare (1 in 100,000) (1). Rudimentary horns can be subclassified into either the communicating or non-communicating type according to whether or not there is a cavity between the uterine cavity and the horn. The majority of rudimentary horns are of the non-communicating type (72-85\%) (2). The majority of uterine rudimentary horns coexist with a homolateral genitourinary tract malformation and are asymptomatic. However, certain rudimentary horns cause infertility, hematometra, dysmenorrhea or endometriosis as gynecological consequences (3). Pregnancy in such a rudimentary horn is uncommon. The present study therefore describes an unexpected horn pregnancy with an invasive mole, which is an extremely rare presentation. The final diagnosis of a non-communicating rudimentary horn with pregnancy was found intraoperatively and the presence of the invasive mole was confirmed by histopathology.

\section{Case report}

A 29-year-old gravidity-3, parity-1, abortion-1, live-1 female patient during a 7-week period of amenorrhea was referred to the Department of Gynecology of Qilu Hospital of Shandong University (Shandong, China) in March 2015, as the patient was found to possess a uterine deformity and the presence of a hydatidiform mole was suspected 3 days earlier. The patient exhibited a normal menstrual cycle prior to this and the uterine aberration had not been identified until the patient underwent a cesarean in October 2011. A positive pregnancy test was shown 1 year prior when the patient exhibited amenorrhea for 36 days. The patient was then diagnosed with an ectopic pregnancy and was treated with methotrexate (MTX $50 \mathrm{mg}$ intramuscularly, every other day, 3 times daily) in The Second Hospital of Shandong University (Shandong, China), followed by being discharged with a decreased level $(57.70 \mathrm{mIU} / \mathrm{ml})$ 
of $\beta$-human chorionic gonadotropin $(\beta$-HCG) 2 weeks later (compared to $634.94 \mathrm{mIU} / \mathrm{ml}$ at the point of admission).

The patient was asymptomatic until amenorrhea occurred for 47 days, and a uterine anomaly and right ovarian cyst was revealed by gynecological ultrasound in The Second Hospital of Shandong University (Shandong, China). Another gynecological ultrasound revealed a high possibility of a rudimentary horn of the uterus, with a solid mass cyst and bilateral ovarian cysts. In addition, the serum $\beta-\mathrm{HCG}$ level was $>200,000 \mathrm{mIU} / \mathrm{ml}$ (normal range, $0-10 \mathrm{mIU} / \mathrm{ml}$ ), without pelvic pain or abnormal vaginal bleeding. The patient was then admitted to Qilu Hospital of Shandong University with a pregnancy in the rudimentary horn of the uterus. Upon general examination, the blood pressure was 113/66 $\mathrm{mmHg}$ (normal range, $90 / 60-120 / 90 \mathrm{mmHg}$ ), with a pulse rate of 89 beats per min (normal range, 60-100 beats per min). The result of the vaginal examination revealed normal external genitalia, and a vagina and single cervix that appeared normal. The size of the uterine body appeared to be that of a 2-month pregnancy. In addition, a mass of $\sim 5 \times 4 \mathrm{~cm}$, with mild tenderness, was palpated on the right side of the uterus. The results of the gynecological ultrasound and the pelvic magnetic resonance imaging (MRI) scan are reported in Figs. 1 and 2. The ultrasound illustrates a suspicious gestational trophoblastic disease in the rudimentary uterine horn, with a rich blood flow signal at the right side of the uterus. Pelvic MRI shows the endometrial thickening and gestational trophoblastic disease in the rudimentary uterine horn. The chest X-ray was negative.

A combined hysteroscopy and laparoscopy was performed 6 days subsequent to admission. The intraoperative findings are shown in Fig. 3. The uterus was increased in size, as is normal for a pregnancy at 2 months, and a normal ovary and fallopian tube was identified on the left side. A rudimentary horn covered with tortuous and expansive blood vessels, measuring $8 \times 6 \times 5 \mathrm{~cm}$, was identified on the right side of the uterus, with a normal ovary and fallopian tube attached. The horn was connected to the right wall of the uterus above the internal orifice of the uterus via a thin fibrous band. The hysteroscopy revealed that the cervix and uterine cavity were normal and that the left tubal opening was clear. However, the right tubal opening could not be observed. The endometrium was thick, presenting as an Arias-Stella reaction. As a result, the rudimentary horn was dissected and diagnostic curettage was performed by combined hysteroscopy and laparoscopy. The ipsilateral adnexa was conserved as it appeared to be normal. The dissected tissues were sent for routine pathological tests (Fig. 4).

Paraffin-embedded, 4- $\mu \mathrm{m}$ thick tissue sections from the dissected tissues were stained with p57 and Ki-67 antibodies for immunohistological analysis. All tissue sections were de-waxed, rehydrated and incubated in $3 \%$ hydrogen peroxide for $10 \mathrm{~min}$ at room temperature to halt endogenous peroxidase activity, then incubated for 30-60 min with an anti-p57 antibody (Mouse Monoclonal Antibody; ready-to-use dilution; cat. no., ZM-0252; ZSGB-BIO, Beijing, China) and anti-Ki67 antibody (Rabbit Monoclonal Primary Antibody; ready-to-use dilution, 2 ug/ml; cat. no., 790-4286; Roche Diagnostics Shanghai Ltd., Shanghai, China) at room temperature. Then the sections were incubated with a HRP-labeled staining reagent (Universal Immuno-peroxidase Polymer Anti-Mouse/Rabbit
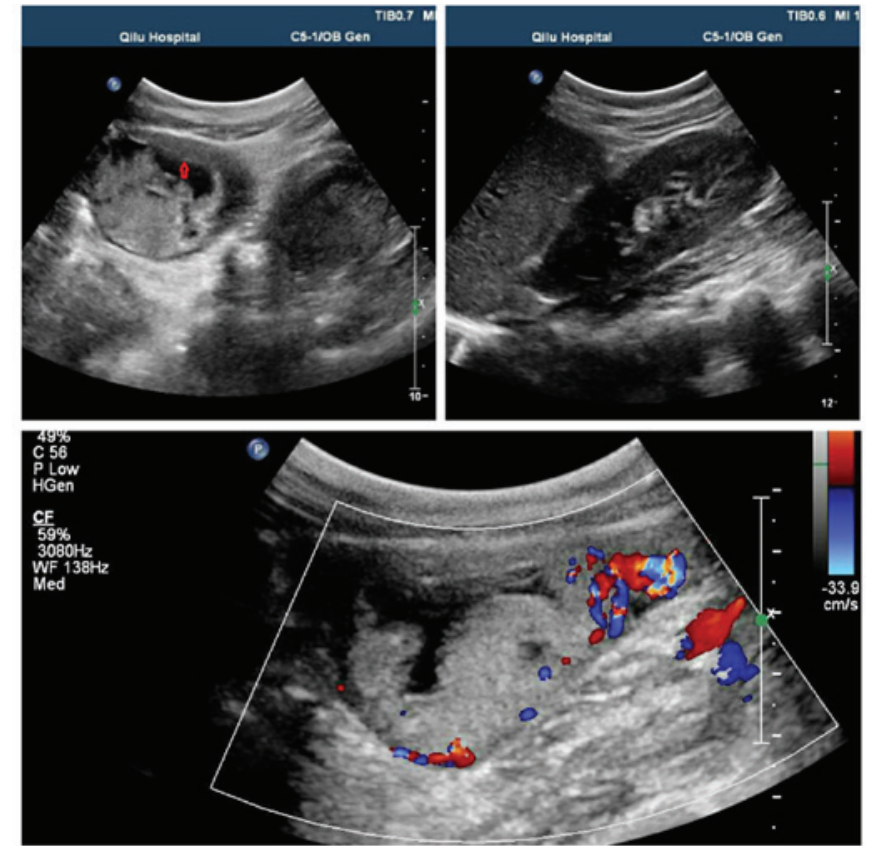

Figure 1. Ultrasound showing a suspicious gestational trophoblastic disease in the rudimentary uterine horn, with a rich blood flow signal at the right side of the uterus. Arrowheads indicate the thickness of the wall of the rudimentary horn.
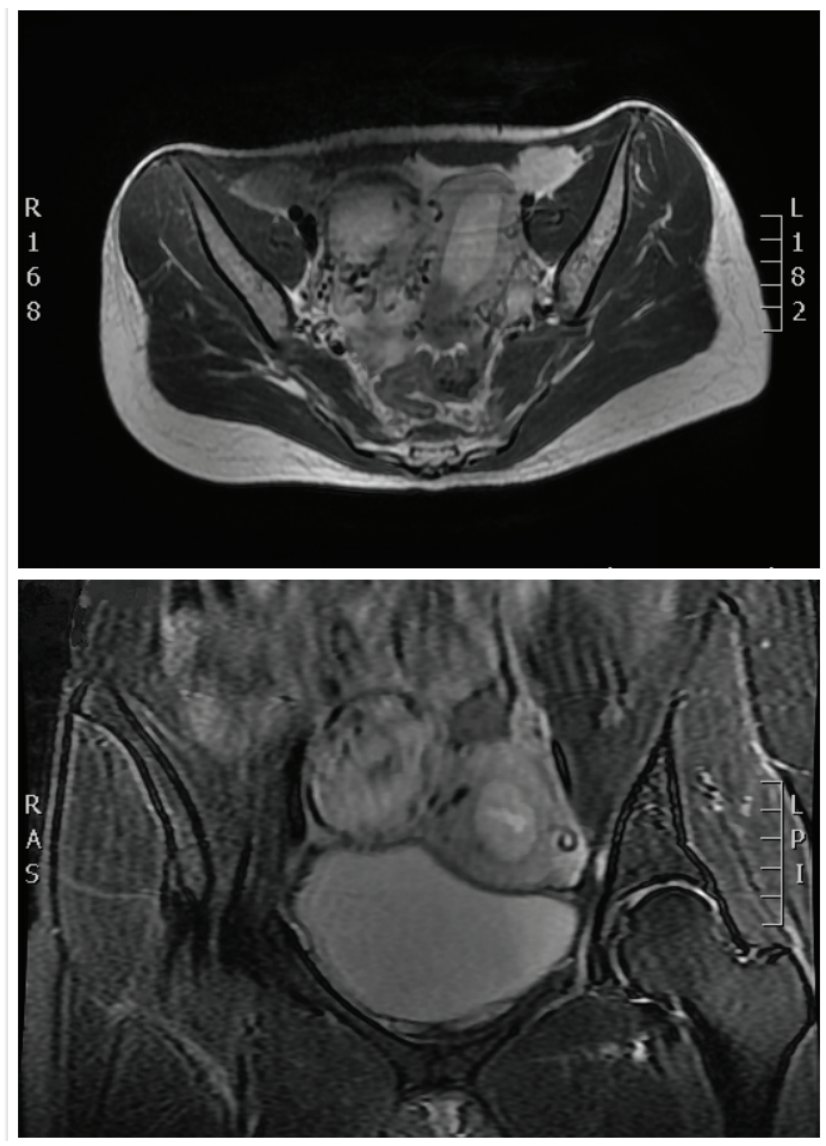

Figure 2. Pelvic magnetic resonance imaging showing the endometrial thickening and gestational trophoblastic disease in the rudimentary uterine horn.

Immunohistochemical Staining reagent; ready-to-use dilution; cat. no., PV-8000; ZSGB-BIO) at room temperature for 
$20 \mathrm{~min}$. Sections were developed with a DAB kit (ZSGB-BIO) used according to the manufacturer's protocol. The tissue sections were washed 3 times with phosphate-buffered saline (ZSGB-BIO) between each step. Following staining, the tissue sections were counterstained using hematoxylin (ZSGB-BIO), and subsequently dehydrated using ethanol and xylene. Permount TM Mounting Medium (ZSGB-BIO) was then applied to the coverslips. An ECLIPSE 80i research microscope (Nikon Corporation, Tokyo, Japan) was used to observe the immunohistological staining results at a magnification of $\mathrm{x} 40-\mathrm{x} 100$.

Histological sections of the right rudimentary horn revealed the myometrial invasion of massive placental villi and metatypical trophoblastic cells (Fig. 4A). Immunohistological staining demonstrated positive expression of p57, and a high Ki-67 proliferation index (Fig. 4A). Diagnostic curettage sections showed the decidualized endometrium (Fig. 4B). Based on the high serum $\beta$-HCG level, imageological results and the final pathology, the patient was diagnosed as follows: invasive hydatidiform mole, rudimentary uterine horn and scarred uterus. Post-operative care and follow-up were performed. Following the surgery, the serum $\beta$-HCG level decreased from $182,232 \mathrm{mIU} / \mathrm{ml}$ to $26,086 \mathrm{mIU} / \mathrm{ml}$. Concurrently, the patient was reluctant to undergo chemotherapy and chose to close follow-up. The patient was monitored with monthly serum $\beta$-HCG level examinations for the first 6 months following the operation, and then every 2 months for the subsequent 6 months. Physical examinations and necessary imaging examination was performed at intervals of 6-12 months. At present, the patient has a good condition.

\section{Discussion}

Uterine rudimentary horns occur following the partial non-development of one Müllerian duct and incomplete fusion with the contralateral side (4). The majority of rudimentary horns are non-communicating with the uterine cavity, and may connect to the lower uterine segment via a thin fibrous band. According to the American Fertility Society classification of Müllerian anomalies (5), the patient in the present study exhibited a non-communicating rudimentary horn of the II-b variety.

Rudimentary horn pregnancy (RHP) is rare clinical condition and represents a form of ectopic gestation, estimated to occur in 1 in 100,000 to 140,000 pregnancies (6). As reported in the study by Pal et al, Mauriceau and Vassal first described pregnancy in a rudimentary horn in 1669 (7). Tsafrir et al outlined the criteria for sonographic diagnoses of RHP (8). As Siwatch et al reported, the sensitivity of ultrasound with respect to the diagnosis of rudimentary horn pregnancies ranges between 29 and $33 \%$ only (9). However, MRI has a major role with respect to the diagnosis of RHP and should be considered when the condition is suspected (8).

Due to the maldevelopment of the musculature and poor distensibility of the wall of the rudimentary horn, the prognosis of RHP is often poor. The condition generally presents as a rupture of the uterine wall between 10 and 15 gestational weeks (10), manifesting as a life-threatening condition of high risk, involving acute abdominal pain with heavy intraperitoneal hemorrhage. Therefore, timely diagnosis and management is

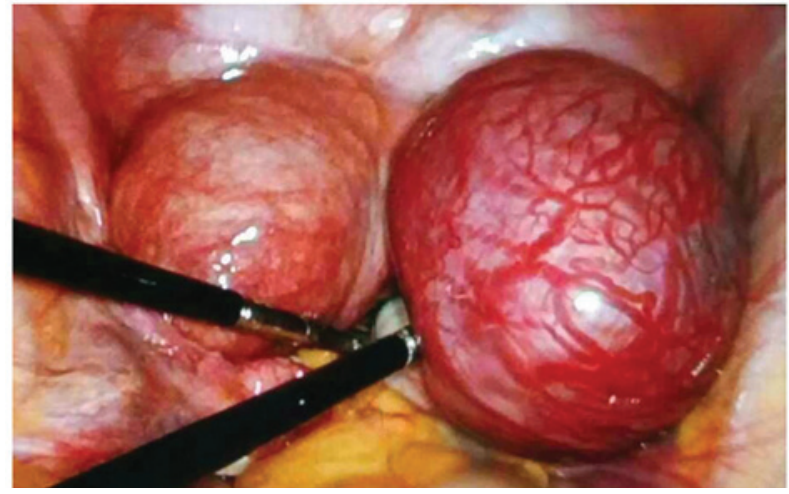

Figure 3. Laparoscopic view at the start of the procedure with a left-sided uterus and a right-sided rudimentary horn containing the pregnant tissue.

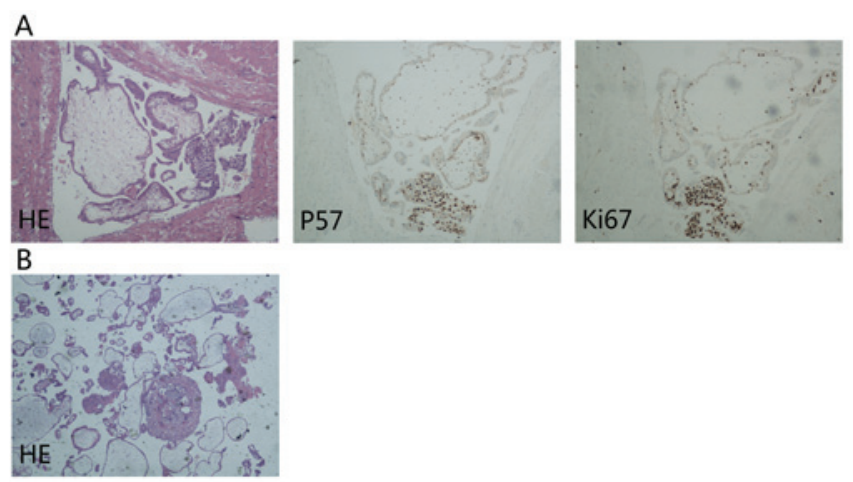

Figure 4. Microscopic findings. (A) Histological sections of the right rudimentary horn revealing the myometrial invasion of massive placental villi and trophoblastic cells, with hematoxylin staining, and p57 and Ki-67 immunostaining (magnification, x100). (B) Diagnostic curettage sections showing decidualized endometrium (magnification, $\mathrm{x} 40$ ). HE, hematoxylin and eosin.

required. However, there are certain patients that reach a near term pregnancy (11).

Gestational trophoblastic neoplasia (GTN) encompasses a group of pregnancy-associated disorders, including invasive hydatidiform moles (IHMs), choriocarcinoma, placental-site trophoblastic tumors and epithelioid trophoblastic tumors. IHM is a benign tumor that occurs following the myometrial invasion of a hydatidiform mole via direct extension through the tissue or venous channels to other areas of the body subsequent to conception. While for the majority of patients IHM commonly occurs less than half a year after the evacuation of a molar pregnancy, the condition may also develop subsequent to any gestation (12). The majority of affected patients present with either vaginal bleeding subsequent to the evacuation of a molar pregnancy, abortion, term delivery or ectopic pregnancy, or with subinvolution of the uterus, abdominal pain or false pregnancy symptoms. The prognosis is good, as IHM mainly causes local invasion of myometrial invasion. A total of 10-17\% of hydatidiform moles will result in invasive moles, of which $\sim 15 \%$ metastasize to the lungs or the vagina (13).

Invasive moles are mostly diagnosed clinically rather than pathologically, based on persistently elevated levels of $\beta$-HCG subsequent to molar evacuation (14). Close monitoring of the serum $\beta$-HCG level and imageological changes are key for the effective diagnosis and management of GTN. The symptoms 
required to diagnose post-molar GTN include at least 1 of the following (15): Levels of $\beta$-HCG that have plateaued for 4 consecutive values over 3 weeks; a $\beta$-HCG level that has risen $10 \%$ across 3 values over 2 weeks; persistent $\beta$-HCG levels 6 months subsequent to molar evacuation; a histopathological diagnosis of choriocarcinoma; or the presence of metastatic disease (16). Characteristic ultrasonographic scans of an invasive mole reveal a hyperechoic mass in the myometrium, with an ill-defined boundary, accompanied by theca lutein ovarian cysts. A color Doppler ultrasound will typically reveal abundant blood flow signals in the presence of GTN (17). A chest X-ray is performed for the diagnosis of lung metastases. If lesions are exhibited on the chest radiograph, brain MRI and whole-body computed tomography (CT) are recommended to exclude the possibility of a more widespread disease. The utility of the new imaging modality of positron emission tomography/CT scans should also be investigated.

The incidence of invasive hydatidiform moles in rudimentary horns is extremely rare. Due to the atypical characteristics of the condition, it is difficult to definitively diagnose it prior to surgery (18).

IHM in a rudimentary horn is uncommon. As the number of gestational weeks increases, the markedly enlarged rudimentary horn can occur adjacent to the normal uterus body, easily leading to misdiagnosis as an intrauterine pregnancy. An early diagnosis of IHM, of which imaging examination is foremost, is essential for the successful management of the condition in order to prevent maternal morbidity and mortality. However, the condition is usually diagnosed subsequent to laparoscopy or laparotomy for an acute abdomen (7).

The excision of the rudimentary horn and an ipsilateral salpingectomy, preferably preserving the ovary, is the recommended treatment for patients hoping to preserve fertility potential, thus preventing uterine rupture, future ectopic pregnancies and dysmenorrhea (1). Although laparotomy has traditionally been performed, with the availability of improved laparoscopic equipment and techniques, laparoscopic surgery has more recently been used to successfully treat several patients (19). The present study described the laparoscopic removal of a rudimentary horn with IHM. In such cases, a harmonic scalpel can be utilized to effectively amputate the uterine horn, whilst ensuring the surgical field is relatively bloodless, thus reducing the length of the surgery and the recovery of the patient. The rudimentary horn of the uterus can be removed via a morcellator or a fetch bag via a minilaparotomy. A round ligament should be fixed in the ipsilateral cornu of the normal section of the uterus in case of uterine torsion.

The prognosis for IHM is excellent due to its inherent sensitivity to chemotherapy. With the adoption of the novel International Federation of Gynecology and Obstetrics stage-risk scoring system (15), a score of 0-6 indicates a low risk of metastasis and a score of $\geq 7$ suggests a high risk. Patients with low-risk metastatic GTN should be treated with single-agent MTX or actinomycin-D chemotherapy, with resulting survival rates approaching $100 \%$ (20). At present, an etoposide, MTX, actinomycin D, cyclophosphamide and vincristine/oncovine chemotherapy regimen is widely used for patients at high risk of developing a GTN, which has resulted in improved remission and survival rates (21). Nevertheless, the point at which to stop chemotherapy that results in highest efficacy and low toxic effects should be better defined. There is no consensus with respect to the proper time point at which to stop chemotherapy subsequent to biochemical remission (12). Generally, chemotherapy should continue at least until the attainment of biochemical remission, and two additional courses should be administered following the confirmation of normal weekly serum $\beta$-HCG levels. Medical treatment with MTX or feticide, and posterior pregnancy rudimentary horn excision by laparoscopy has been proposed by Cutner et al (19), with the aim of shrinking the horn and thus facilitating a less invasive surgery.

Close follow-up should be insisted upon subsequent to the return of the $\beta$-HCG level to normal and the completion of chemotherapy. Patients are monitored with monthly serum $\beta$-HCG level examinations for the first 6 months after the completion of chemotherapy and then every 2 months for the subsequent 6 months. Physical examinations should be performed at intervals of 6-12 months and other diagnostic techniques such as ultrasonographic scans or X-rays are necessary. In addition, the use of contraception is recommended for 12 months subsequent to the completion of chemotherapy to allow for uninterrupted $\beta$-HCG follow-up and to reduce the potential teratogenicity associated with a subsequent pregnancy (22).

In conclusion, IHM in a rudimentary horn is rare, and diagnosis is challenging. In view of the life-threatening condition that follows rupture, Muram et al (23) suggested that when a rudimentary horn of the uterus is identified prior to conception, it should be resected prophylactically. Maintaining vigilant care and obstetric expertise is required to prevent the morbidity associated with this devastating condition. The removal of the uterine rudimentary horn is essential to reduce future risk. However, excision of the ipsilateral adnexa is controversial. In the present study, the ipsilateral adnexa was conserved since it appeared to be normal. Despite the removal of the RHP, the patient should be advised to undergo chemotherapy for a better prognosis, with a close follow-up. The present study highlights the requirement for high clinical suspicion of this rare condition.

\section{References}

1. Chopra S, Keepanasseril A, Rohilla M, Bagga R, Kalra J and Jain V: Obstetric morbidity and the diagnostic dilemma in pregnancy in rudimentary horn: Retrospective analysis. Arch Gynecol Obstet 280: 907-910, 2009.

2. Sevtap HK, Aral AM and Sertac B: An early diagnosis and successful local medical treatment of a rudimentary uterine horn pregnancy: A case report. Arch Gynecol Obstet 275: 297-298, 2007.

3. Lankford, J.C., P. Mancuso and R. Appel: Congenital Reproductive Abnormalities. J Midwifery Womens Health, 2013.

4. Gonçalves E, Prata JP, Ferreira S, Abreu R, Mesquita J, Carvalho A and Pinheiro P: An unexpected near term pregnancy in a rudimentary uterine horn. Case Rep Obstet Gynecol 2013: 307828, 2013.

5. The American Fertility Society classifications of adnexal adhesions, distal tubal occlusion, tubal occlusion secondary to tubal ligation, tubal pregnancies, mullerian anomalies and intrauterine adhesions. Fertil Steril 49: 944-955, 1988

6. Pal SK, Purkait D, Modak G and Dawn CS: Pregnancy in rudimentary horn. J Indian Med Assoc 81: 96, 1983.

7. Pal K, Majumdar S and Mukhopadhyay S: Rupture of rudimentary uterine horn pregnancy at 37 weeks gestation with fetal survival. Arch Gynecol Obstet 274: 325-326, 2006. 
8. Tsafrir A, Rojansky N, Sela HY, Gomori JM and Nadjari M: Rudimentary horn pregnancy: First-trimester prerupture sonographic diagnosis and confirmation by magnetic resonance imaging. J Ultrasound Med 24: 219-223, 2005.

9. Siwatch S, Mehra R, Pandher DK and Huria A: Rudimentary horn pregnancy: A 10-year experience and review of literature. Arch Gynecol Obstet 287: 687-695, 2013.

10. Nahum GG: Rudimentary uterine horn pregnancy. The 20th-century worldwide experience of 588 cases. J Reprod Med 47: 151-163, 2002.

11. Cheng C, Tang W, Zhang L, Luo M, Huang M, Wu X and Wan G: Unruptured pregnancy in a noncommunicating rudimentary horn at 37 weeks with a live fetus: A case report. J Biomed Res 29: 83-86, 2015.

12. Ng TY and Wong LC: Diagnosis and management of gestational trophoblastic neoplasia. Best Pract Res Clin Obstet Gynaecol 17: 893-903, 2003.

13. Lurain JR: Gestational trophoblastic disease I: Epidemiology, pathology, clinical presentation and diagnosis of gestational trophoblastic disease and, management of hydatidiform mole. Am J Obstet Gynecol 203: 531-539, 2010.

14. Lurain JR and Brewer JI: Invasive mole. Semin Oncol 9: 174-180, 1982.

15. Ngan HY, Bender H, Benedet JL, Jones H, Montruccoli GC and Pecorelli S; FIGO Committee on Gynecologic Oncology: Gestational trophoblastic neoplasia, FIGO staging and classification. Int J Gynaecol Obstet 83 (Suppl 1): S175-S177, 2003.

16. Lurain JR: Gestational trophoblastic disease II: Classification and management of gestational trophoblastic neoplasia. Am J Obstet Gynecol 204: 11-18, 2011.
17. Nair $\mathrm{K}$ and Al-Khawari $\mathrm{H}$ : Invasive mole of the uterus-a rare case diagnosed by ultrasound: A case report. Med Ultrason 16: 175-178, 2014.

18. van Esch EM, Lashley EE, Berning B and de Kroon CD: The value of hysteroscopy in the diagnostic approach to a rudimentary horn pregnancy. BMJ Case Rep 2010: pii: bcr0820103229, 2010.

19. Cutner A, Saridogan E, Hart R, Pandya P and Creighton S: Laparoscopic management of pregnancies occurring in non-communicating accessory uterine horns. Eur J Obstet Gynecol Reprod Biol 113: 106-109, 2004.

20. Alazzam M, Tidy J, Hancock BW and Osborne R: First line chemotherapy in low risk gestational trophoblastic neoplasia. Cochrane Database Syst Rev 21: CD007102, 2009.

21. Newlands ES, Mulholland PJ, Holden L, Seckl MJ and Rustin GJ: Etoposide and cisplatin/etoposide, methotrexate, and actinomycin D (EMA) chemotherapy for patients with high-risk gestational trophoblastic tumors refractory to EMA/cyclophosphamide and vincristine chemotherapy and patients presenting with metastatic placental site trophoblastic tumors. J Clin Oncol 18: 854-859, 2000.

22. Garrett LA, Garner EI, Feltmate CM, Goldstein DP and Berkowitz RS: Subsequent pregnancy outcomes in patients with molar pregnancy and persistent gestational trophoblastic neoplasia. J Reprod Med 53: 481-486, 2008.

23. Muram D, McAlister MS, Winer-Muram HT, Smith WC. Asymptomatic rupture of a rudimentary uterine horn. Obstet Gynecol 69: 486-487, 1987. 